\title{
O DIAGNÓSTICO DA AÇÃO DE CONTROLE DA RESOLUÇÃO DE PROBLEMAS MATEMÁTICOS DE PROFESSORES EM FORMAÇÃO
}

\author{
THE DIAGNOSIS OF ACTION TO CONTROL THE SOLUTION OF \\ MATHEMATICAL PROBLEMS OF TEACHERS IN TRAINING \\ PAULO GONÇALO FARIAS GONÇALVES 1 \\ ISAURO BELTRÁN NÚÑEZ²
}

\section{RESUMO}

0 diagnóstico do estado dos conhecimentos e habilidades de estudantes se constitui como um recurso importante para balizar as práticas pedagógicas. Nesse sentido, o presente trabalho tem como objetivo caracterizar o nível de desenvolvimento inicial da orientação da ação de controle da resolução de problemas matemáticos de futuros professores. Realizado por meio de uma prova pedagógica, os dados foram submetidos a uma análise de conteúdo e de correspondência com a orientação adotada como referência. Verificamos uma compreensão do controle como detaIhamento da ação de resolver problemas, indicando uma incompreensão acerca do que é e de como se realiza essa ação. Portanto, é essencial chamar a atenção para uma adequada utilização do diagnóstico no âmbito da atividade de aprendizagem, transcendendo 0 entendimento sobre esse recurso como mero medidor de desempenho e empregando-o na elaboração de um retrato mais amplo acerca do que os discentes sabem, suas dificuldades e seus potenciais.

Palavras-chave: Diagnóstico. Aprendizagem. Controle. Resolução de problemas.

\section{ABSTRACT}

The diagnosis of the state of knowledge and skills of students is an important resource to guide pedagogical practices. In this sense, the present work aims to characterize the level of initial development of the orientation of the action to control the resolution of mathematical problems of future teachers. Performed through a pedagogical test, the data were subjected to content analysis and correspondence with the guidance adopted as a reference. We verified an understanding of control as a detail of the problem solving action, indicating an incomprehension about what it is and how this action is carried out. Therefore, it is essential to call attention to an appropriate use of the diagnosis in the context of the learning activity, transcending the understanding of this resource as a mere performance meter and employing it in the elaboration of a broader picture about what the students know, their difficulties and their potentials.

Keywords: Diagnosis. Learning. Control. Problem solving.

\section{RESUMEN}

El diagnóstico del estado de conocimientos y habilidades de los estudiantes es un recurso importante para orientar las prácticas pedagógicas. En este sentido, el presente trabajo tiene como objetivo caracterizar el nivel de desarrollo inicial de la orientación de la acción para controlar la resolución de problemas matemáticos de los futuros profesores. Realizado mediante una prueba pedagógica, Ios datos obtenidos fueron sometidos a un análisis de contenido y de correspondencia con la orientación adoptada como referencia en la pesquisa. Verificamos una comprensión del control como un detalle de la acción para resolver problemas, indicando una incomprensión sobre qué es esta

1 Doutor em Educação. Professor Adjunto da Universidade Federal do Cariri, Brejo Santo- CE. E-mail: paulo.goncalo@ufca.edu.br. Orcid: https://orcid.org/0000-0001-5714-2008.

2 Doutor em Ciências Pedagógicas. Professor Titular da Universidade Federal do Rio Grande do Norte, Natal - RN. E-mail: isaurobeltran@ yahoo.com.br. Orcid: https://orcid.org/0000-0003-3224-4694. 
acción y cómo se lleva a cabo. Por tanto, es fundamental llamar la atención sobre un uso adecuado del diagnóstico en el contexto de la actividad de aprendizaje, trascendiendo la comprensión de este recurso como mero medidor de desempeño y utilizándolo en la elaboración de un panorama más amplio sobre lo que saben los estudiantes, sus dificultades y sus potenciales.

Palabras clave: Diagnóstico. Aprendizaje. Control. Solución de problemas.

\section{INTRODUÇÃO}

0 diagnóstico do estado dos conhecimentos e habilidades de estudantes de diferentes níveis de escolaridade se constitui como uma importante função das avaliações, tanto em nível nacional, quanto internacional. Avaliações externas realizadas em larga escala como Prova Brasil, o Exame Nacional de Desempenho de Estudantes (ENADE) e o Programme for International Student Assessment (PISA), por exemplo, podem prover informações que auxiliem na constituição de um retrato indicativo da qualidade dos diferentes sistemas educacionais sobre os quais se debruçam e subsidiar seus atores na elaboração de ações que os aperfeiçoem.

Comentando sobre as prováveis causas do baixo desempenho dos discentes mexicanos no PISA, Zilberstein e Olmedo (2017, p. 150) discutem, dentre outros aspectos, o papel dos professores, ao mencionar que:

[...] nem sempre os docentes levam em conta a função diagnóstica da avaliação com um enfoque científico integral, que lhes permita determinar potencialidades e dificuldades nos alunos; basicamente se centram no resultado, quer dizer na classificação ou na medição.

Nesse caso, a ênfase no produto, em detrimento da compreensão do processo pelos professores gera um diagnóstico limitado, amputado de toda a riqueza que envolve a atividade de aprendizagem e de informações que permitam balizar o planejamento de melhorias.

Apontando a relevância de um conhecimento profissional docente que instrumentalize o professor na elaboração do diagnóstico de elementos da representação mental que guiam os estudantes nas atividades, Núñez e Barros (2019, p. 90) sugerem uma prática que promova nos discentes

[...] a possibilidade de elaborar e reelaborar a orientação das ações que devem realizar, permitindo-Ihes planejar, executar, acompanhar e regulá-las como via de assimilação de conteúdos, o que contribui com 0 aprender a aprender e com a independência cognoscitiva. Para tanto, os professores precisam de um conhecimento profissional coerente com o ensino comprometido com uma aprendizagem consciente e autorregulada, na qual os estudantes são protagonistas.

Por conseguinte, é fundamental que os professores vivenciem, durante a formação inicial e continuada, iniciativas que lhes permitam aprender a diagnosticar e a caracterizar conhecimentos e habilidades colocados em ação, considerando a atuação de seus discentes tanto de forma independente, quanto com algum tipo de ajuda de materiais didáticos ou de outro indivíduo.

Diante do quadro apresentado até aqui, o presente artigo, enquanto recorte de Gonçalves (2020), tem como objetivo caracterizar o nível de desenvolvimento inicial da orientação da ação de controle 
da resolução de problemas matemáticos de professores em formação inicial nas áreas de Ciências Naturais e Matemática.

0 trabalho é composto pela seção de introdução, que apresenta uma problematização, justificativa do objeto de estudo e seu objetivo; a fundamentação teórica, que discute uma perspectiva de diagnóstico à luz do Enfoque Histórico-Cultural; a metodologia, que apresenta o contexto, os sujeitos, o instrumento de coleta, os processos de organização e de análise dos dados; os resultados e discussões, que sintetizam a compreensão da ação de controle da resolução de problemas dos alunos segundo a orientação planejada pelo professor; e as considerações finais, que apresentam as conclusões que decorrem do diagnóstico realizado.

\section{O DIAGNÓSTICO NO CONTEXTO DO ENFOQUE HISTÓRICO-CULTURAL}

De maneira geral, o diagnóstico consiste em um momento do processo formativo que permite subsidiar o professor e os alunos na caracterização e compreensão do estado em que encontram-se conhecimentos e habilidades, possibilitando a adequação da atividade de aprendizagem aos potenciais e limitações dos estudantes. Esse momento é focado na coleta de "[...] informações para a avaliação-intervenção, a fim de transformar ou modificar algo nos alunos, de um estágio inicial para um potencial, o que permite uma atenção diferenciada (ZILBERSTEIN; OLMEDO, 2017, p. 157).

No contexto do Enfoque Histórico-Cultural, destacamos dois pesquisadores que trazem subsídios importantes para a discussão do diagnóstico, são eles: L. S. Vygotsky (1896-1934) e P. Ya. Galperin (1902-1988).

Um dos conceitos mais conhecidos da Teoria Histórico-Cultural é a Zona de Desenvolvimento Próximo ${ }^{3}$. Emergindo no âmbito da discussão da relação entre aprendizagem e desenvolvimento, permeados pelas contribuições das relações sociais sobre esses dois processos, Vygotsky (1995) chama de Zona de Desenvolvimento Próximo (ZDP) a diferença entre o conjunto de atividades das quais 0 sujeito consegue empreender sozinho (que se configuram como seu nível de desenvolvimento real) e aquelas atividades que 0 indivíduo consegue realizar, mas apenas com a mediação de outra pessoa.

Essa metáfora empregada por Vygotsky (1995) faz alusão ao potencial de desenvolvimento do ser humano, "[...] graças a ação transformadora do sujeito sobre a realidade em conjunção com a orientação cultural que recebe dos outros [...]" (FARIÑAS, 2009, p. 5). 0 potencial, que se consolida a partir de um movimento dialético entre aprendizagem e desenvolvimento (RICO, 2003), é uma condição necessária para a formação da personalidade.

Uma resultante do conceito de Zona de Desenvolvimento Próximo para a atividade de aprendizagem diz respeito a necessidade do professor atuar não sobre 0 rol de conhecimentos e habilidades que 0 aluno domina (nível de desenvolvimento atual), mas sobre 0 conjunto de conhecimentos e habilidades que 0 estudante ainda não domina, porém, consegue colocá-los em prática por meio de algum nível de ajuda (material didático, ajuda de outro indivíduo, etc.), ou seja, sobre o nível de desenvolvimento potencial.

Atuar no âmbito da ZDP implica primeiramente em realizar o diagnóstico da compreensão do estudante sobre o conteúdo a ser abordado em sala. Uma via para realização desse diagnóstico seria

\footnotetext{
3 Apesar da tradução mais comum ser Zona de Desenvolvimento Proximal, entendemos que o termo "proximal" pode sugerir uma qualificação ao substantivo "zona". Como Vygotsky (1995) discute a ZDP enquanto uma região em que o desenvolvimento se aproxima, é iminente, optamos pela tradução Zona de Desenvolvimento Próximo.
} 
por meio de questionamentos ou situações-problemas que direcionem o estudante a externalizar sua compreensão sobre o conteúdo e ainda colocá-la em prática.

Somente a partir da identificação do nível de desenvolvimento inicial dos discentes é que o docente tem uma parte das informações necessárias para a elaboração e/ou adaptação de um Sistema Didático que atue sobre a ZDP, a fim de que a atividade idealizada e materializada por meio desse sistema seja internalizada e o nível de desenvolvimento dos estudantes seja elevado quantitativamente e qualitativamente.

Debruçando seus estudos sobre a formação de conhecimentos e habilidades na ZDP, P. Ya. Galperin elabora a Teoria de Formação Planejada das Ações Mentais e dos Conceitos enquanto um modelo de explicação do processo de internalização da atividade externa. De forma complementar à L. S. Vygotsky, P. Ya. Galperin elabora um método que operacionalizou, em relação a atividade diagnóstica, a identificação e caracterização do nível de desenvolvimento real.

Outro elemento importante para subsidiar a construção do Sistema Didático é a busca por uma orientação desejada, que se coloque no nível de desenvolvimento potencial dos estudantes.

Segundo Galperin (1979), a orientação é um elemento central da atividade de aprendizagem. Conforme 0 autor, a orientação é posta em ação no momento em que o indivíduo

[...] realiza um exame da situação nova, confirma ou não o significado racional ou funcional dos objetos, prova e modifica a ação, traça um novo caminho e mais adiante, durante o processo de realização, leva a cabo um controle da ação de acordo com as modificações previamente estabelecidas (GALPERIN, 1979, p. 81).

Nesse sentido, ao se colocar em uma situação que necessite resolver e que a solução não seja evidente, o ser humano precisa empreender uma reflexão para organizar uma representação mental que possa subsidiar sua ação de maneira apropriada. É nesse momento que a orientação é mobilizada. Tratando da relevância desse processo mental, Galperin (1992, p. 78) diz que, "[...] a função vital da mente consiste em seu comportamento orientador, baseado na reflexão mental de uma situação problemática".

Assim, a orientação do sujeito implica em uma representação mental que permite planejamento, antevisão, direção da ação, entre outros. Por conta disso, é essa compreensão que norteia o sujeito na execução e fornece as condições para a realização do controle da ação.

Segundo a Teoria de Formação Planejada das Ações Mentais e dos Conceitos, a orientação do indivíduo se dá por meio da Base Orientadora da Ação (BOA), que consiste na "[...] representação antecipada da tarefa, assim como o sistema de orientadores, que são necessários para seu cumprimento, formam o plano da futura ação, a base para sua direção" (GALPERIN, 2001, p. 46). Esse entendimento parte da premissa de que toda a ação humana consciente é precedida por uma base orientadora, determinante para sua qualidade (GALPERIN, 1989a).

Essa imagem antecipada fornece, ainda antes da execução da ação, o conjunto de condições nas quais o indivíduo se apoiará durante sua realização e acompanhamento (TALIZINA, 1988, 2000, 2009).

Além disso, a escolha adequada da Base Orientadora da Ação se constitui, quando internalizada, como uma ação do pensamento; e ainda permite que o sujeito se utilize dela enquanto orientação para outras atividades que estejam dentro dos limites da BOA (GALPERIN; TALIZINA, 1965).

Tendo em vista o papel do professor na organização do processo de ensino, de modo a possibilitar ao estudante uma internalização adequada das ações e dos conceitos presentes no currículo, 
é fundamental que esse profissional formule e materialize o esquema de uma BOA desejável, que corresponda ao produto da orientação idealizada para 0 aluno ao final da aprendizagem. Diante dessa necessidade, Galperin (1989b) apresenta o Esquema da Base Orientadora Completa da Ação (EBOCA).

0 EBOCA pode ser entendido como a representação materializada da Base de Orientadora da Ação desejável para uma determinada ação (GALPERIN, 1989b). Segundo o autor esse esquema:

[...] incorpora tudo o que um aluno deve 'entender' na tarefa à sua frente, e esse 'entendimento' é expresso pela ação: 0 aluno sistematicamente (não a esmo!) executa todas as instruções do esquema corretamente nas tarefas, que variam substancialmente. Esse 'comportamento' indica que 0 aluno está sendo guiado pelas relações essenciais nas tarefas e que suas ações são racionais (GALPERIN, 1989b, p. 70).

0 esquema pode ser elaborado inicialmente pelo professor para que os discentes reelaborem a sua orientação com um conteúdo desejável, de modo a internalizá-lo como produto da aprendizagem.

Para Núñez e Ramalho (2017, p. 13), o Esquema da Base Orientadora Completa da Ação: "[...] fornece aos estudantes uma ferramenta cultural para a generalização teórica, que permite a compreensão de um conjunto de situações ou de um dado domínio". Por meio do EBOCA, os discentes terão à sua disposição desde o princípio da atividade de aprendizagem um guia completo para execução e controle das tarefas.

Um forma de Esquema da Base Orientadora Completa da Ação possível consiste em evidenciar três aspectos inerentes a ação mental que será objeto da atividade de aprendizagem: a definição da ação mental a ser formada (modelo do objeto); o sistema operacional de execução da ação (modelo da ação) e 0 sistema de operações para controlar a execução da ação (modelo de controle).

Embora possuam similaridades e se inter-relacionem, a Base Orientadora da Ação (BOA) e 0 Esquema da Base Orientadora Completa da Ação (EBOCA) não são a mesma coisa. Galperin (1989b) afirma que enquanto o EBOCA é um esquema representado no plano materializado, a BOA se refere ao seu reflexo mental, após um processo de internalização. Ademais, enquanto há uma constância no EBOCA, sua BOA correspondente pode ter variações em seu conteúdo, conforme 0 sujeito empreende alguma atividade de aprendizagem.

No contexto do diagnóstico inicial, o EBOCA é utilizado como referencial, como um espelho de respostas que representa o maior nível de desenvolvimento dos estudantes para os conhecimentos e habilidades analisadas.

Nessa perspectiva, a análise do diagnóstico acontece por meio de uma comparação entre 0 conteúdo desejável, disposto no EBOCA do professor, e as respostas de cada um dos estudantes.

\section{METODOLOGIA}

Esta pesquisa é um recorte de Gonçalves (2020), que empreendeu um estudo do tipo Experiência Formativa. A Experiência Formativa consiste em um dos métodos utilizados dentro do Enfoque Histórico-Cultural para investigação processo de planejamento, diagnóstico, aplicação e avaliação de atividades de aprendizagem. Assim, o presente trabalho terá como enfoque 0 momento de diagnóstico inicial da compreensão dos estudantes acerca da ação de controle da resolução de problemas matemáticos. 
0 contexto investigado foi o Instituto de Formação de Educadores (IFE), campus da Universidade Federal Cariri (UFCA), no município de Brejo Santo, Ceará. Em específico, a pesquisa foi aplicada no componente curricular Laboratório de Educação Matemática I, disciplina obrigatória do $7^{0}$ semestre da Licenciatura em Matemática e optativa para os demais cursos oferecidos no IFE.

No que se refere aos participantes da pesquisa, os sujeitos foram oito alunos matriculados na referida disciplina, ofertada no primeiro semestre acadêmico de 2019, sendo: seis alunos da Licenciatura em Matemática, um da Licenciatura Interdisciplinar em Ciências Naturais e Matemática e uma estudante da Licenciatura em Biologia. Especificamente, são cinco homens e três mulheres, com idades entre 19 e 33 anos. Enquanto três sujeitos não exerciam à docência, cinco participantes possuíam experiência média de 1 ano e 11 meses como professores na Educação Básica, nas áreas de Ciências Naturais, Matemática, Física e Química.

0 instrumento utilizado nessa pesquisa foi a prova pedagógica, que tem 0 intuito de "[...] diagnosticar o estado dos conhecimentos, hábitos e habilidades dos sujeitos em um momento determinado" (CEREZAL; FIALLO, 2005, p. 128). Assim, a prova pedagógica é útil para verificação do nível de desenvolvimento dos participantes, seja no início, no decorrer ou no final do processo formativo.

De modo específico, utilizamos o que León et al. (2002, p. 64) classifica como prova pedagógica de desenvolvimento que "[...] se baseia na capacidade do estudante de elaborar e expressar as ideias com clareza e precisão, de forma oral ou escrita".

Seu potencial na obtenção de dados, que permitam uma caracterização dos níveis de desenvolvimento de cada estudante em relação a suas orientações, justificam a utilização da prova pedagógica no âmbito da pesquisa.

Utilizamos a prova pedagógica de forma individual e sem ajuda, com intuito de verificar a compreensão dos participantes em relação a orientação para resolver problemas matemáticos (modelo do objeto, modelo da ação e modelo de controle) e o entendimento dos estudantes sobre o conceito de controle da resolução de problemas matemáticos.

Para estruturação da referida prova, elaboramos um planejamento, detalhado no Quadro 1:

Quadro 1 - Plano de elaboração da prova pedagógica para diagnóstico inicial.

\begin{tabular}{|l|l|}
\hline \multicolumn{1}{|c|}{ Objetivo } & \multicolumn{1}{c|}{ Pergunta } \\
\hline $\begin{array}{l}\text { Identificar a compreensão do modelo do objeto da } \\
\text { BOA de resolver problemas matemáticos. }\end{array}$ & $\begin{array}{l}\text { - Quando um estudante aprende a resolver problemas em Matemática deve aprender } \\
\text { também a realizar o controle (regulação) da aprendizagem durante esse processo. Em } \\
\text { relação a essa situação, responda: } \\
\text { 1) } 0 \text { que é resolver um problema matemático? }\end{array}$ \\
\hline $\begin{array}{l}\text { Identificar a compreensão do modelo a ação da } \\
\text { BOA de resolver problemas matemáticos. }\end{array}$ & 2) Quais são os passos necessários para se resolver um problema matemático? \\
\hline $\begin{array}{l}\text { Identificar a compreensão acerca do conceito de } \\
\text { controle da resolução de problemas matemáticos. }\end{array}$ & 3) 0 que é controlar (regular) o processo de resolução de problemas matemáticos? \\
\hline $\begin{array}{l}\text { Identificar a compreensão dos estudantes acerca } \\
\text { do modelo de controle da resolução de problemas } \\
\text { matemáticos. }\end{array}$ & $\begin{array}{l}\text { 4) Supondo que durante sua atuação docente precise avaliar como seus alunos reali- } \\
\text { zam o controle (regulação) do processo de resolução de problemas matemáticos. Para } \\
\text { isso, construa uma chave de resposta contendo as etapas para se resolver problemas } \\
\text { (transcrição das respostas da questão 2) e suas respectivas etapas de controle. }\end{array}$ \\
\hline
\end{tabular}

Fonte: Elaborado pelos autores. 
A organização dos dados ocorreu por meio da planilha eletrônica Excel, dispondo cada sujeito da pesquisa em uma linha e suas respectivas respostas às questões da prova pedagógica em colunas.

No que concerne à análise dos dados, adotamos como referência a orientação desejada elaborada por Gonçalves (2020), que versou sobre a compreensão do conceito de resolução de problemas matemáticos e de seu controle, bem como as formas de coloca-los em ação, como apresentado no Quadro 2:

Quadro 2 - Orientação desejável, sistematizada pelo professor.

\begin{tabular}{|c|c|c|}
\hline & Resolução de problemas matemáticos & Controle da resolução de problemas matemáticos \\
\hline Conceito & $\begin{array}{l}\text { Atividade de busca pela solução de uma contradição definida } \\
\text { a partir do que é conhecido e desconhecido em uma situação- } \\
\text {-problema, por meio de conhecimentos matemáticos. }\end{array}$ & $\begin{array}{l}\text { É a verificação da qualidade da execução da atividade de re- } \\
\text { solver problemas matemáticos e correção, quando necessária, } \\
\text { segundo uma orientação determinada. }\end{array}$ \\
\hline Ação & $\begin{array}{l}\text { A1: Analisar a situação-problema } \\
\text { 01: Ler o enunciado e determinar se corresponde a uma situa- } \\
\text { ção-problema. Caso corresponda, resolver utilizando o cartão } \\
\text { de estudo. Do contrário, procurar outra orientação. } \\
\text { 02: Reconhecer o que é conhecido e o que é desconhecido } \\
\text { 03: Definir o problema, a partir da contradição entre o conhe- } \\
\text { cido e o desconhecido } \\
\text { 04: Representar o problema a partir do que é conhecido, des- } \\
\text { conhecido e procurado. } \\
\text { A2: Planejar uma estratégia para solução do problema } \\
\text { 05: Determinar as condições para a solução do problema. } \\
\text { 06: Elaborar uma estratégia para resolução, segundo as condi- } \\
\text { ções do problema } \\
\text { A3: Executar a estratégia para resolução do problema } \\
\text { 07: Realizar a resolução do problema de acordo com o plane- } \\
\text { jado. } \\
\text { 08: Interpretar o resultado encontrado em função do que é } \\
\text { procurado. } \\
\text { A4: Avaliar a resolução do problema } \\
\text { 09: Verificar se a solução do problema está de acordo com o } \\
\text { que é procurado } \\
\text { 010: Caso a resposta esteja correta, refletir sobre os aspectos } \\
\text { que favoreceram a esse resultado } \\
\text { 011: Caso a resposta seja negativa, ou não tenha chegado a } \\
\text { uma resposta, refletir e reformular caso seja necessário, o } \\
\text { problema e/ou a estratégia de solução. Corrigir os erros. } \\
\text { 012: Relacionar os resultados obtidos com conhecimentos } \\
\text { anteriores e com novos conhecimentos. }\end{array}$ & $\begin{array}{l}\text { C1: O enunciado trata de uma situação-problema? } \\
\text { C2: Reconheceu-se o conhecido? Reconheceu-se o desconheci- } \\
\text { do? } \\
\text { C3: Definiu-se o problema, a partir do que é conhecido e } \\
\text { desconhecido na situação-problema? } \\
\text { C4: Foi criado um modelo do problema a ser resolvido? } \\
\text { C5: Analisou-se e determinou-se as condições nas quais se } \\
\text { resolverá o problema? Quais são essas condições? } \\
\text { C6: Por que a estratégia elaborada é apropriada para solução } \\
\text { do problema, segundo as condições estabelecidas? } \\
\text { C7: A solução do problema se realiza segundo o planejado, } \\
\text { considerando as condições e a representação do problema? } \\
\text { C8: Chegou-se a uma solução do problema? Caso positivo, } \\
\text { Analisou-se a resposta de modo a justificá-la como resposta } \\
\text { ao problema? } \\
\text { c9: As operações anteriores estão adequadas ao que é } \\
\text { procurado? } \\
\text { C10: Quais aspectos favoreceram ao resultado? } \\
\text { C11: O que é preciso corrigir para solucionar o problema? } \\
\text { C12: Como os resultados obtidos se relacionam com elemen- } \\
\text { tos já conhecidos e com novos conhecimentos? }\end{array}$ \\
\hline
\end{tabular}

Fonte: Adaptado de Gonçalves (2020).

A orientação desejável foi adotada como uma referência para as respostas dos alunos, que foram classificadas segundo os níveis de convergência: correto (C), parcialmente correto (PC) e ausente $(A)$.

Findada a explanação acerca do percurso metodológico empreendido, discutiremos na próxima seção os resultados da pesquisa. 


\section{RESULTADOS E DISCUSSÕES}

Discutiremos os resultados em função da caracterização das orientações dos alunos em comparação com cada elemento presente na orientação planejada pelo professor (Quadro 2).

Na questão 1, foi solicitado aos respondentes explicitar uma definição do que é resolver problemas matemáticos. A partir dessa análise e comparação, apresentamos os resultados sintetizados no Quadro 3.

Quadro 3 - Análise das respostas segundo modelo do objeto da orientação do professor.

\begin{tabular}{|c|c|c|c|c|c|}
\hline \multirow{2}{*}{ Participante } & \multicolumn{4}{|c|}{ Definição de resolução de problemas matemáticos } & \multirow{2}{*}{$\begin{array}{c}\text { Percentual correto } \\
\text { por aluno }\end{array}$} \\
\cline { 2 - 5 } & Atividade & $\begin{array}{c}\text { Busca pela } \\
\text { solução }\end{array}$ & Contradição & $\begin{array}{c}\text { Conhecimentos } \\
\text { matemáticos }\end{array}$ & A \\
\hline P1 & A & C & A & A & $25 \%$ \\
\hline P2 & A & C & A & A & C \\
\hline P3 & A & C & A & C & $50 \%$ \\
\hline P4 & A & A & A & A & $0 \%$ \\
\hline P5 & A & A & A & A & $0 \%$ \\
\hline P6 & A & A & A & A & $0 \%$ \\
\hline P7 & A & C & A & A & $25 \%$ \\
\hline P8 & A & C & A & C & $50 \%$ \\
\hline $\begin{array}{c}\text { Percentual correto } \\
\text { da turma }\end{array}$ & 0\% & $62,5 \%$ & $0 \%$ & $25 \%$ & \\
\hline
\end{tabular}

Fonte: Elaborado pelos autores.

Verificamos que a maioria das respostas dos discentes (62,5\%), apresentaram a definição do que é resolver problemas matemáticos enquanto um processo de busca pela solução, enquanto que $25 \%$ mencionaram 0 uso de conhecimentos matemáticos durante esse processo. Por outro lado, nenhuma das respostas explicitou a atividade ou a contradição entre aquilo que 0 aluno sabe (conhecido) e o que ele não sabe (desconhecido) como aspectos da resolução de problemas.

No que se refere aos níveis de similaridade com o modelo do objeto contido na orientação do professor, dois alunos atingiram 50\%, três $25 \%$ e outros três discentes apresentaram compreensões sobre 0 que é resolver problemas matemáticos incompatíveis com a referência utilizada.

Esse resultado comunga com outros diagnósticos dos níveis de desenvolvimento de diferentes habilidades gerais como: interpretar gráficos (PEREIRA, 2013), modelar cientificamente (ANDRADE, 2017), explicar propriedades físicas das substâncias e dos materiais (NÚÑEZ; RAMALHO, 2018), classificar (NÚÑEZ, 2018), escrever textos argumentativos em Química (ARAÚJO, 2018), tomar decisões (NÚÑEZ; RAMALHO, 2019) e realizar uma leitura crítica (FAÇANHA, 2019).

Em todos os casos, a compreensão dos estudantes e, em particular, o modelo do objeto dessas orientações concentraram-se nos menores níveis de desenvolvimento dos objetos, em relação às orientações adotadas como referência por cada uma das investigações mencionadas.

A questão 2 indagou os estudantes sobre como resolver problemas matemáticos. 0 Quadro 4 apresenta uma síntese dos resultados obtidos: 
Quadro 4 - Análise das respostas em função do modelo da ação da orientação do professor.

\begin{tabular}{|c|c|c|c|c|c|c|c|c|c|c|c|c|c|}
\hline \multirow{2}{*}{ Participante } & \multicolumn{4}{|c|}{ A1 } & \multicolumn{2}{|c|}{ A2 } & \multicolumn{2}{|c|}{ A3 } & \multicolumn{4}{|c|}{ A4 } & \multirow{2}{*}{$\begin{array}{c}\text { Percentual } \\
\text { correto por aluno }\end{array}$} \\
\hline & 01 & 02 & 03 & 04 & 05 & 06 & 07 & 08 & 09 & 010 & 011 & 012 & \\
\hline P1 & A & A & C & A & A & $\mathrm{C}$ & C & A & $\mathrm{C}$ & A & $C$ & A & $33,3 \%$ \\
\hline $\mathrm{P} 2$ & $A$ & A & $C$ & $A$ & $A$ & $C$ & $C$ & $A$ & $C$ & $A$ & $A$ & A & $33,3 \%$ \\
\hline P3 & A & A & C & A & A & A & C & A & A & A & A & A & $16,7 \%$ \\
\hline P4 & A & $A$ & A & A & $A$ & A & $C$ & A & $A$ & $A$ & A & A & $8,3 \%$ \\
\hline P5 & $A$ & A & C & A & $\mathrm{A}$ & $\mathrm{C}$ & $C$ & A & $\mathrm{C}$ & A & A & A & $33,3 \%$ \\
\hline P6 & $C$ & $\mathrm{C}$ & $C$ & $C$ & $A$ & $A$ & $C$ & A & $A$ & A & A & A & $41,7 \%$ \\
\hline P7 & A & A & C & $A$ & A & A & C & A & $\mathrm{C}$ & $A$ & A & A & $25 \%$ \\
\hline P8 & A & A & $C$ & A & $A$ & $C$ & $C$ & A & $A$ & $A$ & A & A & $25 \%$ \\
\hline $\begin{array}{c}\text { Percentual } \\
\text { correto da turma }\end{array}$ & 12,5 & 12,5 & 87,5 & 12,5 & 0 & 50 & 100 & 0 & 50 & 0 & 12,5 & 0 & \\
\hline
\end{tabular}

Fonte: Elaborado pelos autores.

Verificamos que as operações de maiores recorrência foram: "07: Realizar a resolução do problema de acordo com o planejado" (100\%) e "03: Definir o problema, a partir da contradição entre 0 conhecido e o desconhecido" (87,5\%). Esse fato sugere uma compreensão dos estudantes da necessidade de haver previamente uma clareza sobre o que é solicitado no problema antes de colocar em prática o processo de resolução.

Por outro lado, há quatro operações inseridas nos momentos de orientação (05: Determinar as condições para a solução do problema.), execução (08: Interpretar o resultado encontrado em função do que é procurado.) e controle (010: Caso a resposta esteja correta, refletir sobre os aspectos que favoreceram a esse resultado; 012: Relacionar os resultados obtidos com conhecimentos anteriores e com novos conhecimentos.) que não foram citadas por nenhum dos respondentes, o que sugere que, interpretando a compreensão dos discentes segundo a orientação do professor, em todos os momentos da atividade de resolver problemas matemáticos há ações constituídas por sistemas de operações incompletos, o que torna a compreensão dessa habilidade limitada.

Acrescentando o fato de que quatro operações só tiveram uma recorrência $(12,5 \%)$ e duas operações com duas recorrências (50\%), notamos um distanciamento na compreensão do modelo da ação da orientação de resolver problemas matemáticos dos participantes da pesquisa com a orientação planejada pelo professor.

Outro aspecto importante foi em relação às operações corretas apresentadas pelos alunos. Enquanto a resposta de maior similaridade com a orientação do professor teve $41,7 \%$ de operações corretas, três tiveram $33,3 \%$ e duas $25 \%$. Além disso, um participante atingiu 16,7\% e um apenas $8,3 \%$.

De modo similar outras investigações apontam, no diagnóstico de uma determinada habilidade geral estudada, um domínio incompleto do sistema de ações e operações que constituem as orientações desejadas.

Em Pereira (2013), o autor identifica que apenas cinco das quatorze operações que constituem a orientação de interpretar gráficos planejada pelo pesquisador, são explicitadas pelos participantes da pesquisa, enquanto que seis operações estiveram presente nas BOAs dos discentes. Fato similar 
ocorre nos trabalhos de Núñez e Ramalho (2018) e Façanha (2019), que mostram ausência de respostas corretas em três e duas operações, respectivamente.

Apresentando uma análise das orientações dos discentes, segundo dimensões da modelação científica, Andrade (2017) verifica que as respostas mais recorrentes são inadequadas, "[...] mesmo sabendo que essas dimensões fazem parte do conhecimento disciplinar do licenciando para ensinar modelagem científica" (ANDRADE, 2017, p. 195).

0 entendimento sobre 0 que é 0 controle da resolução de problemas matemáticos foi 0 objeto da questão 3 da prova pedagógica. Partindo da orientação desejável, detalhada no Quadro 2, verificamos que nenhum dos discentes apresenta uma resposta que se aproxima da definição de controle da resolução de problemas matemáticos.

Passando a se debruçar sobre as respostas dos participantes para melhor entender como eles compreendem o conceito, podemos dividi-las em três tipos, são eles: ações diversas como sinônimo de controle na resolução de problemas matemáticos $(50 \%)$, o controle enquanto uma ação metódica (25\%) e respostas tautológicas $(25 \%)$.

No primeiro grupo de respostas (50\%), os participantes definem o controle na resolução de problemas matemáticos como sinônimos de ações diversas como comandar, administrar, impor rigor, regular e direcionar; todas associadas ao processo de solução de problemas.

Entre os discentes que compreendem o controle enquanto uma ação metódica (25\%), apresentamos, no Quadro 5, as respostas apresentadas:

Quadro 5 - 0 controle enquanto uma ação metódica.

\footnotetext{
“É agir de forma metódica e reflexiva, ou seja, delimitar métodos para a resolução do problema, testá-los seguindo as etapas escolhidas. Caso o método aproxime-se da resolução, seguir os próximos. Caso contrário, identificar o erro, e buscar um outro método. Acredito que essa é uma função do professor". (Participante P1).

"Saber utilizar os métodos de resolução de problemas de uma forma sequencial para se obter os resultados corretos" (Participante P4).
}

Fonte: Elaborado pelos autores.

Podemos observar que 0 controle seria esse elemento balizador da utilização de métodos de solução de problemas, norteando esse processo visando a obtenção de resultados apropriados.

Convém destacar que, em uma das respostas, o participante P1 ainda acrescenta uma qualidade, atribuindo ao controle um aspecto reflexivo, que permite uma tomada de decisão em função da resposta obtida. Esse aspecto também é evidenciado por González-Moreno (2017) como um dos indicadores de modificações nas estratégias autorregulatórias empreendidas pelos participantes de seu experimento formativo. Para a autora, ao possibilitar que 0 indivíduo repense suas ações, a reflexão se coloca como importante subprocesso, em particular, da ação de controle.

A questão quatro solicita quais são as operações necessárias para 0 controle da resolução de problemas matemáticos, a partir de uma transcrição do modelo da ação da BOA já descrita pelos alunos. Assim como no resultado obtido sobre o conceito de controle, nenhum dos estudantes apresenta uma compreensão sobre a ação de controle da resolução de problemas matemáticos convergente com a orientação desejada (Quadro 2). 


\section{Analisando as respostas dos discentes, apresentamos no Quadro 6 o modelo de controle pre-} sente na orientação de cada um desses participantes:

\section{Quadro 6 - Modelo de controle da BOA inicial dos alunos.}

\section{Aluno P1:}

1- Ler 0 enunciado da questão.

2- Verificar 0 que se está pedindo.

3- Conhecer os métodos disponíveis para se conseguir resolver o problema.

4- De forma racional, testá-los, realizando as operações matemáticas.

5- Sempre observar os erros e acertos, para as próximas tomadas de decisões. 6- Eliminar as etapas que não permitiram 0 acerto.

- OBS: Identificar se há algum "problema cognitivo" que impeça a resolução.

Aluno P2:

- Leitura atenta da situação que está sendo apresentada, identificando o que está sendo perguntado.

- Mentalmente buscamos no nosso repertório cognitivo problemas semelhantes a esse e como o resolvemos. Quando não temos ou não lembramos tentamos buscar conhecimentos que estejam relacionados com o tema.

- Nessa etapa se busca uma forma de se resolver o problema, o que pode ser um caminho já conhecido ou não.

\section{Aluno P3:}

- Ler 0 enunciado atentamente, dando total importância as informações apresentadas na questão.

- Sabendo do que se trata o problema é importante ter ciência sobre quais as conexões entre a sentença e os saberes necessários para a solução.

- Elaborar a resposta através da sua perícia com as ferramentas necessárias.

- Finalizar a ação encontrando a determinação almejada.

\section{Aluno P4:}

- Maturidade adquirida ao longo da vida escolar;

- A partir da maturidade adquirida no ambiente escolar 0 aluno conseguirá resolver de forma correta.

\section{Aluno P5:}

1. Leitura do problema. Identificação dos dados (ou variáveis) e condições. Reconhecer o comando.

2. Reconhecer proximidade do problema com algum conteúdo matemático. Identificar se esse conteúdo (técnica, etc.) atende ao que o problema pede.

3. Criar plano de partida com a técnica escolhida (2). Verificar se ela leva ao que o problema direciona. Se não, reformular o plano ou técnica.

4. Visualizar se o resultado obtido é pertinente ao que se procura. Se sim, conclui se. Se não, rever etapas (2) e (3).

Aluno P6:

Fazer como que os alunos vejam os dados que o problema tem e com isso fazer eles raciocinarem qual é o melhor jeito de se trabalhar com o problema.

Observar como os alunos acharam as respostas dos problema.

Mostrar como esses problemas sena na vida dele.

Aluno P7:

1) Realizar uma boa leitura, anotando os dados.

2) Identificar o problema e o que da Matemática que posso utilizar para solucioná-lo.

3) Compreender se realmente a solução encontrada faz parte do problema, ou se, não está se equivocando.

Aluno P8:

- Através da reflexão absorvendo o máximo de informações;

- Perceber e associar os conteúdos estudados com o problema;

- Tendo como base toda as estratégias, aplicá-las de forma prática no problema.

Fonte: Elaborado pelos autores. 
De maneira geral, observamos que a compreensão dos discentes acerca do modelo de controle reduz esse componente da orientação inicial a um detalhamento do modelo da ação.

Esse fato, associado ao resultado apresentado pelos discentes à resposta sobre o conceito de controle da resolução de problemas matemáticos, indica que os participantes da pesquisa não possuem uma compreensão adequada acerca da ação de controle da resolução de problemas.

De modo semelhante, ao diagnosticar o modelo de controle da habilidade de escrever textos argumentativos, Araújo (2018) também encontra um alto percentual (69\%) de operações de controle ausentes entre os discentes.

Esse fato evidencia uma atividade orientadora com deficiências relativas ao controle. A mesma problemática foi apontada por Rico (2002) e Rico e Silvester (2002), que identificaram em suas pesquisas que uma orientação inadequada pode levar a execuções prematuras e a ausência de ações de controle.

Analisando 0 diagnóstico de modo geral, é possível notar que não há uma correlação entre a compreensão dos estudantes sobre 0 que é e como resolver problemas matemáticos e sobre 0 controle desse processo. Em particular, nenhum dos alunos evidenciou, no diagnóstico inicial, ter consciência sobre a realização da ação controle na resolução de problemas matemáticos. Esse fato endossa a afirmação de que a habilidade de controle na resolução de problemas matemáticos dos alunos é pouco desenvolvida, considerando o nível educacional que eles se situam.

\section{CONSIDERAÇÕES FINAIS}

Constituindo-se como uma função de grande relevância para a avaliação, o diagnóstico é, no contexto do Enfoque Histórico-Cultural, um meio para se caracterizar a Zona de Desenvolvimento Próximo dos sujeitos da atividade de aprendizagem.

Considerando a relevância desse processo, empreendemos uma caracterização do nível real da orientação da ação de controle da resolução de problemas matemáticos de uma turma de professores de Ciências Naturais e Matemática em formação.

Em relação ao nível de desenvolvimento inicial dos participantes da pesquisa acerca da orientação da ação de controle da resolução de problemas matemáticos, o fato de ter encontrado baixos níveis de convergências com a orientação planejada pelo professor sugere que os licenciandos participantes não possuíam uma compreensão apropriada, sobretudo, relacionada ao modelo de controle.

Nesse sentido, endossamos um quadro já apontado por outras pesquisas sobre o tema e alertamos para a necessidade de um maior esforço no campo educacional para proposição de práticas educativas focadas em promover melhorias nas ações mentais dos discentes necessárias para 0 aprender a aprender.

Dentre as possibilidades de novos estudos que possam avançar os resultados obtidos por esta pesquisa, um dos caminhos é a realização de investigações que se debrucem também sobre o nível de desenvolvimento potencial dos participantes, adotando a construção de provas pedagógicas com diferentes níveis de ajuda, a fim de caracterizar além do que os sujeitos conseguem realizar sozinhos, aquilo que fazem com algum tipo de auxílio.

Portanto, é essencial chamar a atenção para uma adequada utilização do diagnóstico no âmbito da atividade de aprendizagem, transcendendo a compreensão desse recurso como mero medidor de desempenho e empregando-o na elaboração de um retrato mais amplo acerca do que os discentes sabem, suas dificuldades e seus potenciais. 


\section{REFERÊNCIAS}

ANDRADE, M. A. de. A Base Orientadora da Ação em modelagem científica de licenciandos em Química: um conhecimento disciplinar profissional para o ensino. 2017. 229f. Dissertação (Mestrado em Educação) - Universidade Federal do Rio Grande do Norte, Natal, 2017.

ARAÚJO, M. S. de. Um estudo sobre a reconfiguração da orientação para a ação escrever textos argumentativos em química. 2018. 182f. Tese (Doutorado em Ensino de Ciências e Matemática) - Universidade Federal do Rio Grande do Norte, Natal, 2018.

CEREZAL, J. M.; FIALLO, J. R. ¿Cómo investigar em Pedagogía? La Habana: Pueblo y Educación, 2005, 161f.

FAÇANHA, A. A. de B. A orientação da ação para leitura crítica a partir de uma experiência formativa: uma contribuição para 0 ensino de Química. 2019. 245f. Tese (Doutorado em Educação) - Universidade Federal do Rio Grande do Norte, Natal, 2019.

FARIÑAS, G. L. El enfoque histórico cultural en el estudio del desarrollo humano: para una praxis humanista. Revista Electrónica Actualidades Investigativas en Educación, San Pedro de Montes de 0ca, v. 9, p. 1- 23, nov. , 2009.

GALPERIN, P. Y. Introduccíon a la psicologia: um enfoque dialéctico. Madrid: Pablo del Río Editor, 1979, $160 f$.

GALPERIN, P. Y. Mental Actions as a Basis for the Formation of Thoughts and Images. Soviet Psychology, v. 27, n. 3, p. 45-64, 1989a.

GALPERIN, P. Y. Organization of Mental Activity and the Effectiveness of Learning. Soviet Psychology, v. 27, n. 3, p. $65-82,1989$ b.

GALPERIN, P. Y. Tipos de orientación y tipos de formación de las acciones y de los conceptos. In: QUINTANAR, L. R. (Org.) La formacion de las funciones psicológicas durante el desarrollo del niño. Tlaxcala: Universidad Autónoma de Tlaxcala, 2001, p. 41- 44.

GALPERIN, P. Y. Stage-by-Stage Formation as a Method of Psychological Investigation. Journal of Russian and East European Psychology, v. 4, n. 30, p. 60- 80, 1992.

GALPERIN, P. Y.; TALIZINA, N. F. Control of the Learning Process is Basic. Russian Education and Society, v. 7, n. 12, p. 19-24, 1965.

GONÇALVES, P. G. F. A orientação da ação de controle na resolução de problemas matemáticos em professores: uma experiência formativa à luz da Teoria de P. Ya. Galperin. 2020. 205f. Tese (Doutorado em Educação) - Universidade Federal do Rio Grande do Norte, Natal, 2020.

GONZÁLEZ-MORENO, C. X. Efectos de la enseñanza en la autorregulación del aprendizaje de conceptos científicos en estudiantes universitários. Summa Psicológica, Santiago, v. 14, n. 2, p. 1-13, 2017.

LEÓN, I. N. de.; et al. Metodología de la investigación educacional: Segunda parte. La Habana: Editorial Pueblo y Educación, 2002. 
NÚÑEZ, I. B.; BARROS, S. C. B. de. 0 conhecimento de professores sobre a orientação do estudante na aprendizagem. Cadernos de Pesquisa, São Luís, v. 26, n. 2, p. 87-105, abr./jun. , 2019.

NúÑEZ, I. B.; RAMALHO, B. L. A teoria da Formação Planejada das Ações Mentais e dos Conceitos de P. Ya. Galperin: contribuições para a Didática Desenvolvimental. Obutchénie, Uberlândia, v. 1, n. 1, p. 1- 29, jan./jun. , 2017.

NÚÑEZ, I. B.; RAMALHO, B. L. Diagnóstico do nível de desenvolvimento da orientação de uma ação, em Química Geral, com futuros professores: contribuições da Teoria de P. Ya. Galperin. Obutchénie, Uberlândia, v. 2, n. 2, p. 412-439, maio./ago., 2018.

NÚÑEZ, I. B.; RAMALHO, B. L. Reelaboração da base orientadora da ação na formação de uma habilidade geral: uma experiência na formação de professores de Ciências. In: ALMEIDA, J. J. P. de; DANTAS FILHO, F. F. (Orgs.). Itinerários de pesquisas em Ensino de Ciências e Educação Matemática. Campina Grande: EDUEPB, v. 1, 2019, p. 225-252.

NÚÑEZ, I. B. 0 diagnóstico dos níveis de orientação da ação classificar: contribuição da teoria de P. Ya. Galperin. In: FEITOSA, R. A.; SILVA, S. A. da (Orgs.). Metodologias emergentes na pesquisa em ensino de ciências. Porto Alegre: Fi, 2018, p. 157-175.

PEREIRA, J. E. Formação da habilidade de interpretar gráficos cartesianos em licenciandos em Química segundo a Teoria de P. Ya. Galperin. 2013. 333f. Tese (Doutorado em Educação) - Universidade Federal do Rio Grande do Norte, Natal, 2013.

RICO, P. M. Algunas características de la actividad de aprendizaje y del desarrollo de los alunos. In: GARCÍA, G. B. (Org.). Compendio de Pedagogía. Havana: Pueblo y Educación, 2002, p. 61-67.

RIC0, P. M. La Zona de Desarrollo Próximo (ZDP): Procedimientos y Tareas de Aprendizaje. Havana: Pueblo y Educación, 2003, $94 f$

RICO, P. M.; SILVESTRE, M. O. Proceso de enseñanza aprendizaje. In: GARCÍA, G. B. (Org.). Compendio de Pedagogía. Havana: Pueblo y Educación, 2002, p. 68-79.

TALIZINA, N. F. Psicologia de la enseñanza. Moscou: Editorial Progresso, 1988, $346 f$.

TALIZINA, N. F. Manual de Psicología Pedagógica. México: Universidad Autónoma de San Luís Potosí, 2000, 329f.

TALIZINA, N. F. La teoría de la actividad aplicada a la enseñanza. Puebla: Benemérita Universidad Autónoma de Puebla, 2009, $412 f$.

VYGOTSKY, L. S. Obras Escogidas III (Incluye Problemas del desarrollo de la psique). Madrid: Visor, tomo III, 1995, $383 f$.

ZILBERSTEIN, J. T.; OLMEDO, S. C. Concepción del diagnóstico: ¿por qué y para qué desde posiciones de la didáctica desarrolladora? Obutchénie, Uberlândia, v. 1, n. 1, p. 148-167, jan. /abr., 2017.

RECEBIDO EM: 04 jan. 2021.

CONCLUIDO EM: 21 mai. 2021. 